\title{
Theory of the Trojan-Horse Method - From the Original Idea to Actual Applications
}

\author{
Stefan Typel ${ }^{1,2, \star}$ \\ ${ }^{1}$ Institut für Kernphysik, Technische Universität Darmstadt, Schlossgartenstraße 9, D-64289 Darmstadt, \\ Germany \\ ${ }^{2}$ GSI Helmholtzzentrum für Schwerionenforschung GmbH, Planckstraße 1, D-64291 Darmstadt, Germany
}

\begin{abstract}
The origin and the main features of the Trojan-horse (TH) method are delineated starting with the original idea of Gerhard Baur. Basic theoretical considerations, general experimental conditions and possible problems are discussed. Significant steps in experimental studies towards the implementation of the TH method and the development of the theoretical description are presented. This lead to the successful application of the TH approach by Claudio Spitaleri and his group to determine low-energy cross section that are relevant for astrophysics. An outlook with possible developments in the future are given.
\end{abstract}

\section{Introduction}

Cross sections of low-energy nuclear reactions are crucial ingredients in astrophysical models that describe the nucleosynthesis during Big Bang and stellar evolution, see, e.g., Ref. [1]. They have to be known with sufficient accuracy to predict the abunduncies of the produced elements. Because charged particles participate in the reactions, the cross sections are strongly suppressed by the Coulomb barrier at the low thermal energies of the astrophysical environment. Their direct measurement in the relevant range of energies, the Gamov window, is extremely difficult due to the low reaction rates and many experimental challenges. As an alternative, indirect methods have been proposed to extract the wanted cross sections from suitable surrogate reactions. An example is the Coulomb dissociation of nuclei into fragments with virtual photons to study the inverse radiative capture reactions $[2,3]$.

A particular indirect method that is applicable to astrophysical rearrangement reactions of the type

$$
A+x \rightarrow C+c
$$

was introduced by Gerhard Baur as a theoretical concept in 1986 [4]. In fact, he had suggested the idea already in his invited talk on 'Breakup processes in nuclear reactions' at the 1985 Varna International Summer School on Nuclear Physics [5]. Instead of measuring the cross section of reaction (1) at low energies directly in the laboratory, the breakup reaction

$$
A+a \rightarrow C+c+b
$$

^e-mail: s.typel@gsi.de 

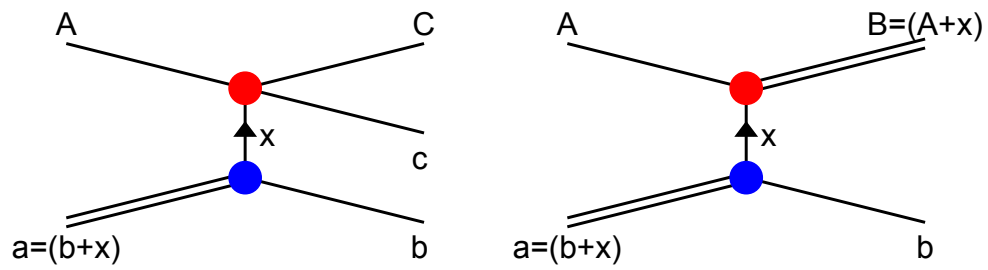

Figure 1. Diagrammatic representation of a transfer reaction to the continuum of the subsystem $C+c$ (left) and to a bound state of the subsystem $B$ (right) with a Trojan-horse nucleus $a$ and a spectator nucleus $b$.

is studied with energies in the initial state above the Coulomb barrier, see the diagram in the left panel of figure 1. The nucleus $a$ is assumed to be well described as a bound state composed of two nuclear clusters $b$ and $x$. Reaction (1) can be considered as a sub-process of (2) where $x$ is the transfered particle and $b$ acts as a spectator. Nucleus $x$ is hidden inside $a$ and brought across the Coulomb barrier to induce the reaction with $A$. This description clearly justifies to call nucleus $a$ the Trojan horse, thus explaining the name of the method. It goes back to Homer's epic poems on the Trojan War and subsequent events. Actually, the capture of Troy with the subterfuge using the Trojan horse is

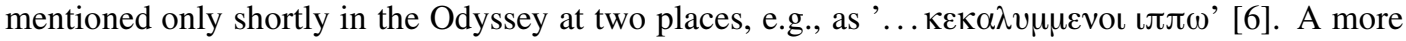
detailed account of the story can be found in Virgil's Aeneid [7].

In order to extract the cross section of the astrophysically relevant reaction (1) from the reaction (2) measured in the laboratory, direct reaction theory is employed. Since the TH reaction is surface dominated, it is expected that the suppression of the cross section of the subprocess, which is astrophysically relevant, by the Coulomb barrier is reduced. Also electron screening will play no role due to the relatively high energies in the initial $A+a$ system. In the original proposal of Gerhard Baur, it was argued that the Fermi motion of $x$ inside the TH $a$ compensates the relative motion of $x$ with respect to $A$ and thus small relative energies in the $A+x$ system were accessible. This corresponds to exploring the tail of the $x+b$ wave function of relative motion in momentum space. However, in the actual application of the TH method by Claudio Spitaleri and his group it was realized that the reaction (2) has to be studied as a quasifree scattering process to exploit the virtues of the TH method. This corresponds to kinematical conditions covering the maximum and not the tail of the momentum distribution.

\section{Theory of direct reactions}

For a sucessfull application of the THM it is essential to utilise direct reaction theory to connect the cross sections of the TH reaction (2), depicted diagrammatically in the left part of figure 1, with that of the astrophysical reaction (1). The general cross section of a reaction with three particles in the final state can be written in nonrelativistic kinematics as

$$
d \sigma=\frac{2 \pi}{\hbar} \frac{\mu_{A a}}{p_{A a}} \frac{d^{3} p_{B b}}{(2 \pi \hbar)^{3}} \frac{d^{3} p_{C c}}{(2 \pi \hbar)^{3}}\left|T_{f i}\right|^{2} \delta\left(E_{B b}+E_{C c}-E_{A a}-Q\right)
$$

where $B$ denotes the $C+c$ system and

$$
Q=\left(m_{A}+m_{a}-m_{C}-m_{c}-m_{b}\right) c^{2}
$$

is the $\mathrm{Q}$ value of the THM reaction (2). The mass of a system $i$ is denoted by $m_{i}$ and the relative momentum between $i$ and $j$ is represented by $\vec{p}_{i j}=\mu_{i j}\left(\vec{p}_{i} / m_{i}-\vec{p}_{j} / m_{j}\right)$ with the reduced mass $\mu_{i j}=$ 
$m_{i} m_{j} /\left(m_{i}+m_{j}\right)$. Actual cross sections that are measured are obtained from (3) by an integration over unobserved quantities taking the energy conservation, expressed by the $\delta$ function, into account.

The essential information on the reaction process is contained in the T-matrix element $T_{f i}$ that is given in post formulation by

$$
T_{f i}=\left\langle\phi_{B} \phi_{b} \exp \left(i \vec{p}_{B b} \cdot \vec{r}_{B b} / \hbar\right)\left|V_{B b}\right| \Psi_{A a}^{(+)}\right\rangle
$$

with the full scattering wave function $\Psi_{A a}^{(+)}$in the initial state and the interaction $V_{B b}$ between the spectator $b$ and the subsystem $B=C+c$ in the final state. The wave function $\phi_{B}$ is the full scattering wave function $\Psi_{C c}^{(-)}$of the $C+c$ system containing information on the scattering matrix elements of the reaction (1) and thus the cross section. If the final state of the the THM reaction was not a transfer to a continuum state $B=C+c$ but to a bound state $B=A+x$, depicted diagrammatically in the right panel of figure 1, the wave function $\phi_{B}$ would contain information on the spectroscopic factors or asymptotic normalisation coefficients of the nucleus $B$. The exact calculation of the T-matrix element (5) is practically impossible and approximations have to be introduced. Several choices are introduced frequently in the literature, e.g., the distorted-wave Born approximation (DWBA), the distorted-wave impulse approximation (DWIA), or the plane-wave impulse approximation (PWIA).

The fully differential cross section of the TH reaction (2) assumes a particular simple form if the PWIA is applied. In this case it can be written in a factorized form as

$$
\frac{d^{3}}{d E_{C c} d \Omega_{C c} d \Omega_{(C c) b}}=K\left|\Phi_{a}(\vec{Q})\right|^{2} \frac{d \sigma^{\mathrm{HOES}}}{d \Omega_{C c}}
$$

with a kinematic factor $K$, and two factors that can be related to the two poles in the diagram of transfer reaction to the continuum, see the left panel of figure 1 . The momentum distribution $\left|\Phi_{a}(\vec{Q})\right|^{2}$ of the transfered particle $x$ inside the TH $a$ depends on the momentum transfer $\vec{Q}$ to the spectator $b$ and is derived from the ground state wave function of relative motion in momentum space $\Phi_{a}$. This factor corresponds to the lower (blue) pole in the diagram. The last factor in eq. (6) is the differential cross section for the astrophysical reaction (1), however, not the wanted on-shell one but a so-called half-offshell cross section. The difference is mainly caused by two facts: (1) the transfered particle $x$ is not on the energy shell, because the standard dispersion relation between its energy and momentum does not apply, (2) the suppression by the Coulomb barrier is reduced as compared to the on-shell cross section. This last feature makes the THM very attractive because it allows to reach extremely low energies in the reaction with reasonable cross sections in contrast to the original astrophysical reaction (1). The actual form of the cross section $\frac{d \sigma^{\mathrm{HOES}}}{d \Omega_{C \mathrm{C}}}$ and its relation to the cross section of reaction (1) depends on the approximations made in deriving the factorized form (6) of the TH cross section. E.g., applying the PWIA and the surface approximation, these cross sections differ by a simple penetrability factor depending on Coulomb wave functions at a certain radius. In less drastic approximations this relation might change leading to a modification of the energy dependence of the extracted astrophysical cross section.

\section{Conditions and problems}

The THM can be realised successfully in experiments if several precondition are met. First of all, a suitable TH nucleus $a$ has to be selected that is well clustered. Typically, a nucleus like ${ }^{2} \mathrm{H}$ or ${ }^{6} \mathrm{Li}$ is chosen in order to be able to study reactions induced by neutrons, protons, deuterons or $\alpha$ particles. The momentum distributions $\left|\Phi_{a}(\vec{Q})\right|^{2}$ of these Trojan horses are well known and peak at zero momentum because they are described as s-wave ground states in cluster models. The kinematic 
conditions for the TH reaction also have to be selected carefully. On the one hand quasifree scattering condition have to be satisfied, corresponding to a zero momentum transfer to the spectator for the above-mentioned Trojan horses, and on the other hand the beam energy in the initial state should match the accessible range of energies of the astrophysical reaction. The separation of the quasifree reaction from other reaction mechanism, e.g., sequential processes, has to be checked experimentally.

In the analysis of a TH experiment, the factorization of the cross section as in eq. (6) is usually presupposed to be valid, however the quality of this approximation needs to be tested in improved theoretical calculations in the future. The relation of the half-off-shell cross section $\frac{d \sigma^{\text {HOES }}}{d \Omega_{C c}}$ to the wanted on-shell cross section of the reaction (1) used in present applications of the THM has to be validated in refined approaches. The extracted cross sections have to be normalized to directly measured data at high energies, which are usually available, because the approximations applied in the derivation of the cross section (6) do not allow to obtain absolute values.

\section{Experimental studies and applications of the TH method}

The kinematic conditions for TH experiments can be realised easily at the Laboratori Nazionali del Sud (LNS) in Catania, e.g., with the Tandem accelarator providing beams with energies of a few $\mathrm{MeV} /$ nucleon, and at similar 'small-scale' reseach laboratories. In addition, the expertise of Claudio Spitaleri and his group matches perfectly with those required to perform successful TH experiments. They studied momentum distributions of clustered nuclei extensively [8,9] and performed tests of the cross section factorization in PWIA [10]. Furthermore, many experiments investigating quasifree scattering and reactions were conducted [11-17]. Hence it is not a surprise that Claudio Spitaleri became interested in the TH method.

The first experimental attempts to apply the TH method to astrophysically important reactions started in the second half of the 1980s [18-21] but it remained unclear how to relate the extracted half-off-shell cross section $\frac{d \sigma^{\mathrm{HOES}}}{d \Omega_{C c}}$ to the actual astrophysical cross section. This situation triggered detailed theoretical considerations in Refs. [22, 23] extending the previous formulation by Gerhard Baur and simulations of actual experiments were performed. The application of the 'surface approximation' lead to an explicit form of the penetrability correction that proved to be decisive in the further development of the TH method. Extensive experimental applications of the TH method followed, see, e.g., the early studies [24-26], and realistic cross sections of astrophysically relevant reactions were obtained.

\section{Further developments and outlook}

Nowadays, the standard application of the TH method concerns non-resonant rearrangement reactions. But the scope of applications was extended to include the study of resonance properties, low-energy elastic scattering, or electron screening effects. The experimental progress is nicely summarized in the recent review [27]. The theoretical development including other indirect methods is discussed in the review [3]. For a further refinement of the TH method, more dedicated high-precision experiments are needed as well as more detailed calculations in order to check the validity of the approximations and the accuracy of the extracted cross sections of astrophysically relevant reactions. E.g., the explicit propagation of the transfered off-shell cluster $x$ can be considered in an extended theoretical description of the TH reaction, leading to a two-step process [28] instead of the one-step description (5). Nevertheless, the final expression of the cross section assumes a similar product form as in eq. (6). A comparison of different theoretical approaches will be very worthwhile. On the experimental side, the 
use of TH nuclei $a$ with clusters in a p-wave ground state and a maximum of the corresponding momentum distribution at nonzero momentum could broaden the range of applications of the TH method even further.

\section{References}

[1] E. G. Adelberger et al., Rev. Mod. Phys. 83, 195 (2011)

[2] G. Baur, C. A. Bertulani and H. Rebel, Nucl. Phys. A 458, 188 (1986)

[3] R. E. Tribble et al., Rept. Prog. Phys. 77, 106901 (2014)

[4] G. Baur, Phys. Lett. B 178, 135 (1986)

[5] G. Baur, Nuclear Energy 25, 183 (1987)

[6] Homer, Odyssey VIII, 503

[7] Virgil, Aeneid II, $13 \mathrm{ff}$.

[8] S. Barbarino et al., Phys. Rev. C 21, 104 (1980)

[9] M. Lattuada et al., Nuovo Cim. A 63, 530 (1981)

[10] P. G. Fallica et al., Phys. Rev. C 24, 1394 (1981)

[11] M. Lattuada et al., Phys. Rev. C 26, 1330 (1982)

[12] M. Lattuada et al., Phys. Rev. C 30, 531 (1984)

[13] M. Lattuada et al., Nucl. Phys. A 458, 493 (1986)

[14] M. Zadro et al., Nucl. Phys. A 474, 373 (1987)

[15] M. Zadro et al., Phys. Rev. C 40, 181 (1989)

[16] G. Calvi et al., Phys. Rev. C 41, 1848 (1990)

[17] S. Blagus et al., Phys. Rev. C 44, 325 (1991)

[18] S. Cherubini et al., Ap. J. 457, 855 (1996)

[19] G. Calvi et al., Nucl. Phys. A 621, 139c (1997)

[20] C. Spitaleri et al., Phys. Rev. C 60, 055802 (1999)

[21] M. Aliotta et al., Eur. Phys. J. A 9, 435 (2000)

[22] S. Typel and H. H. Wolter, Few-Body Systems 29, 75 (2000)

[23] S. Typel and G. Baur, Annals Phys. 305, 228 (2003)

[24] C. Spitaleri et al., Phys. Rev. C 63, 055801 (2001)

[25] A. Musumarra et al., Phys. Rev. C 64, 068801 (2001)

[26] M. G. Pellegriti et al., Nucl. Phys. A 688, 543c (2001)

[27] C. Spitaleri et al., Eur. Phys. J. A 52, 77 (2016)

[28] C. A. Bertulani, M. S. Hussein and S. Typel, arXiv:1707.04563 [nucl-th] 
